\title{
Immunization coverage and predictive factors for complete and age-appropriate vaccination among preschoolers in Athens, Greece: a cross- sectional study
}

Ioanna D Pavlopoulou ${ }^{1 *}$, Koralia A Michail ${ }^{2+}$, Evangelia Samoli ${ }^{3+}$, George Tsiftis ${ }^{4}$ and Konstantinos Tsoumakas ${ }^{1}$

\begin{abstract}
Background: In Greece, several new childhood vaccines were introduced recently but were reimbursed gradually and at different time points. The aim of this study was to assess immunization coverage and identify factors influencing complete and age-appropriate vaccination among children attending public nurseries in the municipal district of Athens.

Methods: A cross-sectional study, using stratified sampling was performed. Immunization history was obtained from vaccination booklets. Demographic and socioeconomic data were obtained from school registries and telephone interviews. Vaccination rates were estimated by sample weighted proportions while associations between complete and age-appropriate immunization and potential determinants by logistic regression analysis.

Results: A total of 731 children (mean age: 46, median: 48, range: 10-65 months) were included. Overall immunization coverage with traditional vaccines (DTP, polio, Hib, HBV, 1st dose MMR) was satisfactory, exceeding $90 \%$, but the administration of booster doses was delayed (range: 33.7- 97.4\%, at 60 months of age). Complete vaccination rates were lower for new vaccines (Men C, PCV7, varicella, hepatitis A), ranging between 61-92\%. In addition, a significant delay in timely administration of Men C, PCV7, as well as HBV was noted $(22.9 \%, 16.0 \%$ and $27.7 \%$ at 12 months of age, respectively). Child's age was strongly associated with incomplete vaccination with all vaccines $(p<0.001)$, while as immigrant status was a predictor of incomplete $(p=0.034)$ and delayed vaccination $(p<0.001)$ with traditional vaccines. Increasing household size and higher maternal education were negatively associated with the receipt of all and newly licensed vaccines, respectively $(p=0.035)$.
\end{abstract}

Conclusions: Our findings highlight the need to monitor uptake of new vaccines and improve age- appropriate administration of booster doses as well as early vaccination against hepatitis B. Immigrant status, increased household size and high maternal education may warrant targeted intervention.

Keywords: Vaccination coverage, Age-appropriate immunization, Predictive factors, Conjugated pneumococcal vaccine, Conjugated meningococcal C vaccine, Varicella vaccine, Greece

\footnotetext{
* Correspondence: idpavlop@yahoo.gr

${ }^{\dagger}$ Equal contributors

"Pediatric Clinic, "P \& A Kyriakou" Children's Hospital, National and

Kapodistrian University of Athens, Faculty of Nursing, Athens, Greece

Full list of author information is available at the end of the article
} 


\section{Background}

Immunization is one of the most effective public health tools, leading to reduced infant and childhood morbidity and mortality, caused by vaccine preventable diseases worldwide [1-4]. However, outbreaks of vaccine preventable diseases continue to occur in developed countries, and this may be attributed to unimmunized or under-immunized subpopulations despite high overall vaccination coverage [5-9]. Several underlying factors for suboptimal vaccine uptake have been described, but are complex and present diversities among different countries, and population groups [10-16]. It is, therefore, essential to monitor vaccination coverage regularly and identify reasons that contribute to delay or non-vaccination at local levels, in order to develop appropriate strategies to address the needs of susceptible populations.

In Greece, vaccines included in the National Immunization Programme (NIP) are universally recommended and are provided free of charge to all resident children, including immigrants. Children can be vaccinated in the private sector or primary health centers and health insurance clinics, the latter two at no additional cost. Vaccinations are recorded in health booklets, but there is no routine nationwide monitoring and no official vaccine reminder system.

As in most developed countries, the Hellenic immunization schedule has become more complex in the last decade, with the introduction of new vaccines and recommendation of more doses of existing vaccines. It is noteworthy that reimbursement of new vaccines has occurred recently, mostly in a gradual and nonsimultaneous process. Therefore, compliance with newly recommended vaccines and associated factors has not been well documented.

Several local and one nationwide study assessing vaccination coverage with old vaccines (DTP/DTaP, OPV/IPV, MMR, Hib and hepatitis B) in pre- school and first grade grammar school children have been published in our country but have not addressed factors influencing under immunization [17-20]. According to a national survey performed in 2006 among 6- year olds, various socioeconomic factors, rather than parental attitudes and beliefs towards vaccines, were identified as predictors for incomplete and delayed immunizations [21]. Since then, new multivalent vaccines, as well as conjugated meningococcal C (Men C), 7-valent pneumococcal (PCV7), varicella (Var) and hepatitis A vaccines (HAV), licensed between 1999 and 2004, were gradually incorporated in the Hellenic NIP. Men C and PCV7 were partially reimbursed in 2005, Var in 2006, and all others, including HAV, were fully compensated in 2008. Moreover, a significant increase in the immigrant population, including children, originating from countries with limited vaccine resources, has occurred during the last years $[22,23]$. At present, data concerning immunization uptake of newer vaccines and related determinants remain insufficient.

The aim of this study was to assess immunization coverage and identify factors affecting complete and age-appropriate vaccination among a large population of children attending public nurseries in the municipal district of Athens, after the introduction of a full reimbursement policy of new vaccines.

\section{Methods}

A cross-sectional study was undertaken using stratified sample design in all public nurseries of the Municipality of Athens, Greece during the school year 2010-2011. The above area consists of 98 nursery schools with a total of 5,401 registered pupils of middle and low income families and is further divided into seven subregions. Nurseries were equally stratified into all seven municipal subregions. These were further subdivided by the child's gender into boys and girls in order to better reflect the children's gender distribution by region. On the day of population recording, a total of 4,165 pupils were present, but only 3,399 were considered for further evaluation, those whose parents had presented a health booklet. The minimum required sample size was estimated, using Snedecor \& Cochran's formula for random sampling from finite populations [24]. Final sample consisted of 731 children (368 boys and 363 girls) with $90 \%$ power and $+/-10 \%$ estimate of true proportion.

Children were randomly sampled from each stratum, as opposed to the entire 3,399 children, in order to extract the number of subjects estimated by sample stratification, followed by personal telephone interviews from children's guardians. In the case of incorrect phone number or non-reply, following a total of five repeated attempts, simple random sampling was repeated, in order to complete the originally calculated number of 731 individuals. The sampling stopped once the minimum sample size was achieved.

All school teachers invited to participate, were informed through interactive sessions organized by the investigators at the beginning of the school year. Following an explanatory letter, all parents/guardians of children of the selected schools were asked to take part in the study by presenting their child's vaccination booklet and by answering to a subsequent telephone interview.

\section{Data collection}

A structured questionnaire completed by the investigators was used. Basic demographic data were collected from school registries on the day of school visit. Detailed vaccination history and use of combination 
vaccines were obtained from vaccination booklets. Parental/guardian attitudes towards immunization and additional information were gathered on a second occasion by telephone interview.

\section{Definitions of vaccination status}

Children were considered completely and timely immunized according to the 2006 NIP guidelines (see Additional file 1: Table S1). Definitions of complete and age- appropriate immunization are presented in Table 1 . We decided to use the 2006 NIP as reference because the majority of the pupils belonged to this age group. Assessment of immunization coverage against hepatitis A was performed separately, since this vaccine was introduced and fully reimbursed in 2008, for all infants older than 12 months.

\section{Ethical considerations}

The study protocol was approved by the ethics committee of the University of Athens, Faculty of Nursing and the review board of the Municipal Nursery of Athens. Informed consent was implied when parents provided their child's health booklet and participated in the telephone interview that followed.

\section{Statistical analysis}

In order to examine associations between complete and age-appropriate vaccination with potential determinants we, initially, applied simple cross-tabulations and the $\mathrm{X}^{2}$-test. Weighted proportions were used to account for the sampling design.

Consequently, we applied multiple logistic regression models [25] including the child's actual age at interview (in years, continuously), father's nationality (Greek versus other), mother's education (high school or less versus college/university), number of children in the family (categorically as: 1, 2, 3 or more), use of multivalent vaccines (3- or 4-valent versus 5 - or 6-valent). Additional variables included the impact of vaccine cost and the insurance coverage on the decision for vaccination

Table 1 Definitions of complete and age- appropriate vaccination

\begin{tabular}{|c|c|c|c|c|}
\hline \multirow[t]{2}{*}{ Vaccine } & \multirow[t]{2}{*}{ Complete vaccination } & \multicolumn{3}{|c|}{ Age appropriate vaccination at the age of } \\
\hline & & 12 months & 24 months & 60 months \\
\hline & 3 doses by the age of 12 months, or & & & \\
\hline \multirow[t]{3}{*}{ DTP/DTaP } & 4 doses by the age of 24 months, or & 3 doses & 4 doses & 5 doses \\
\hline & 5 doses by the age of 60 months & & & \\
\hline & 2 or 3 doses by the age of 12 months plus 1 dose $\geq 12$ months, or & & & \\
\hline \multirow[t]{2}{*}{ OPV/IPV } & 3 doses by the age of 24 months or & 3 doses & 3 or 4 doses & 4 or 5 doses \\
\hline & 4 or 5 doses by the age of 60 months & & & \\
\hline \multirow{4}{*}{ Hib } & 2 or 3 doses by the age of 12 months plus & & & \\
\hline & 1 dose between $\geq 12-23$ months, or & 2 or 3 doces & $>3$ deses & $>3$ deses \\
\hline & 2 doses between $12-24$ months, or & noses & $=5$ uoses & $=5$ uoses \\
\hline & 1 dose at the age of $\geq 24$ months. & & & \\
\hline \multirow{2}{*}{ MMR } & 1 dose between $\geq 12<48$ months, or & \multirow{2}{*}{-} & \multirow{2}{*}{$\geq 1$ dose } & \multirow{2}{*}{2 doses } \\
\hline & 2 doses between $\geq 48-60$ months & & & \\
\hline $\mathrm{HBV}$ & $\geq 3$ doses & 3 doses & 3 doses & 3 doses \\
\hline Men C & $\begin{array}{l}2 \text { doses }<12 \text { months, or } \\
1 \text { dose } \geq 12 \text { months }\end{array}$ & 2 or 3 doses & $\geq 3$ doses & $\geq 3$ doses \\
\hline \multirow{5}{*}{ PCV7 } & $\begin{array}{l}3 \text { doses by the age of } 12 \text { months, plus } \\
1 \text { dose } \geq 12-23 \text { months, or }\end{array}$ & \multirow{5}{*}{3 doses } & \multirow{5}{*}{4 doses } & \multirow{5}{*}{4 doses } \\
\hline & 2 doses from the age of $7-12$ months, plus & & & \\
\hline & 1 dose $\geq 12-23$ months, or & & & \\
\hline & 2 doses between $12-23$ months, or & & & \\
\hline & 1 dose $\geq 24$ months & & & \\
\hline Var & $\begin{array}{l}1 \text { dose between } \geq 12<48 \text { months, or } \\
2 \text { doses between } \geq 48-60 \text { months }\end{array}$ & - & $\geq 1$ dose & 2 doses \\
\hline HAV & $\geq 2$ doses at $\geq 12$ months & - & 2 doses & 2 doses \\
\hline
\end{tabular}

DTP/DTaP: diphtheria, tetanus, pertussis/ acellular pertussis vaccine; OPV/IPV: live attenuated/inactivated polio vaccine; HBV: hepatitis B vaccine; MMR: measles, mumps, rubella vaccine; Hib: haemophilus influenzae type b vaccine; Men C: conjugated meningococcal C vaccine; PCV7: conjugated pneumococcal 7-valent vaccine; Var: varicella vaccine; HAV: hepatitis A vaccine. 
by the parents (both as yes versus no), place of vaccination (private pediatrician versus public health clinic/ insurance doctor) and finally the social security [Social Security Institute (IKA) versus other]. The choice of the variables entered in the models was based on the results from an initial exploratory analysis, possible confounding effects and likelihood ratio backward selection. Special attention was paid in order not to include confounders that were highly correlated (as indicated from the univariate analysis through either $\mathrm{X}^{2}$ test or the Spearman correlation coefficient). Possible determinants of interest, such as the impact of vaccines' cost on the decision for vaccination, were entered in the models irrespectively of statistical criteria. For comparability reasons, in the interpretation of the associations we applied the same model when considering complete or age- appropriate vaccination. When analyzing vaccination status for the three newer vaccines, the influence of vaccine's cost was not considered as a possible determinant since there was no variability as parents of all age appropriate vaccinated children responded that it did not affect their decision. Statistical analysis was performed by SPSS for Windows, version 16.0 (SPSS, Chicago, IL, USA).

\section{Results}

From October 2010 through November 2011, information concerning vaccination history was obtained from a total of 3,399 health booklets available on the day of school visit (participation rate $81.6 \%$ ). The final stratified sample consisted of 731 children, and the response rate was $85 \%$. The mean age of the individuals on the day of sampling was 46 months (median, 48 months; range: 10-65 months).

Table 2 illustrates selected demographic characteristics of the study population. Of the 731 pupils, 504 (68.9\%) were of Greek nationality, 126 (17.2\%) were Albanian, and the remaining 101 (13.8\%) originated from other foreign countries. The majority of children (61\%) were insured with Social Security Institute (IKA). Interestingly, a relatively high proportion of mothers (41\%) were college or university graduates.

Table 3 shows parental attitudes towards vaccines. Nearly two thirds of the parents decided to vaccinate their child in order to offer protection or to prevent disease. Most parents (66.6\%) decided to vaccinate their children at insurance or public health clinics. Although most guardians denied that vaccine cost could be a drawback (91.7\%), a high proportion (66.4\%) admitted that insurance reimbursement would have a positive impact on their decision to vaccinate their child.

\section{Immunization coverage}

Observed sample sizes and weighted proportions for both complete and age- appropriate immunization coverage are presented in Table 4. Complete immunization rates for
Table 2 Selected demographic characteristics of the sample

\begin{tabular}{ll}
\hline & N (\%) \\
\hline Gender & $368(51.7)$ \\
Female & $363(48.3)$ \\
Actual age, (months) & \\
$\leq 24$ & $27(3.7)$ \\
$25-48$ & $363(50.3)$ \\
$49-65$ & $341(45.9)$ \\
Father's nationality & \\
Greek & $504(68.9)$ \\
Albanian & $126(17.2)$ \\
Other immigrant & $101(13.8)$ \\
Number of children & \\
1 & $183(14.7)$ \\
2 & $417(57.4)$ \\
$\geq 3$ & $131(17.9)$ \\
Type of insurance & \\
IKA & $452(61.1)$ \\
Other & $279(38.9)$ \\
Maternal education* & \\
High school or less & $433(59)$ \\
College/University & $297(41.1)$ \\
\hline KA: Socia Secuity Instite &
\end{tabular}

IKA: Social Security Institute; * One mother deceased.

diphtheria, tetanus, pertussis, poliomyelitis, Hib and hepatitis B were satisfactory overall, exceeding 94\%. However, complete overall coverage for MMR was lower, reaching $63.7 \%$. Likewise, age- appropriate immunization rates for the aforementioned vaccines were satisfactory at the age of 12 months, except for hepatitis B, but gradually decreased thereafter. Thus, hepatitis B vaccine uptake did not exceed $27.7 \%$ at 12 months but increased with age reaching $86.1 \%$ at 24 and $97.4 \%$ at 60 months of age.

Among children eligible for varicella vaccination, 435 [61.1\%; 95\% confidence interval (CI): 57.5\%- 64.7\%] were completely immunized, whereas 671 (91.9\%; 95\% CI: 89.9\%-93.9\%) were fully immunized with Men C, 537 (73.1\%; 95\% CI: 69.9\%-76.3\%) with PCV7 and 450 (62\%; 95\% CI: 60.7\%- 67.7\%) with HAV. In contrast, age- appropriate immunization with the above vaccines was significantly lower, ranging between 4 and $71 \%$, the lowest rates being observed among those vaccines that were gradually reimbursed (Men C and PCV7) when compared to the ones that were compensated simultaneously to their implementation (Var, HAV).

Finally, a total of 414 (56.9\%) children (95\% CI: 53.3\%$60.5 \%$ ) were fully immunized with all five traditional vaccines (DTP/DTaP, polio/IPV, Hib, HBV and MMR), 
Table 3 Parental attitudes towards vaccines

\begin{tabular}{ll}
\hline & N (\%) \\
\hline Reasons for vaccinating & $354(47.9)$ \\
Protection, immunity, good health & $162(22.6)$ \\
Prevention of infections & $103(14.4)$ \\
Pediatrician's advice & $112(15.1)$ \\
It is compulsory & \\
Would vaccine cost influence your decision to vaccinate your child? & $75(10.3)$ \\
Yes & $21(2.9)$ \\
Probably yes & $118(15.9)$ \\
Maybe & $56(7.7)$ \\
Probably not & $461(63.3)$ \\
No & \\
Does insurance reimbursement influence your decision? & $487(66.4)$ \\
Yes & $244(33.6)$ \\
No & \\
Where do you vaccinate you child? & $239(33.4)$ \\
Private pediatrician* & $485(65.7)$ \\
Insurance doctor or clinict & $7(0.9)$ \\
Public health clinict &
\end{tabular}

* Doctor's fee only, +No additional costs.

whereas 327 (44.2\%) children (95\% CI: 40.2\%- 47.8\%) were completely immunized with all three recently introduced vaccines (PCV7, Men C, Var).

\section{Multiple logistic regression analysis}

Table 5 presents multiple logistic regression- derived odds ratios (ORs) and corresponding 95\% CIs for the association between sample characteristics and complete and age- appropriate immunization for all five traditional vaccines (DTP/DTaP, IPV/OPV, Hib, MMR and HBV). Factors negatively associated with complete immunization coverage with all of the above vaccines, were the child's increasing age $(\mathrm{p}<0.001)$ and foreign ethnicity $(\mathrm{p}=0.034)$. In addition, ethnicity seemed to play a significant role on age- appropriate vaccination since Greek children had higher odds to be vaccinated at the age of 24 months than immigrant children (OR: 2.57; 95\% CI: 1.71-3.87, $\mathrm{p}<0.001)$. Moreover, age- appropriate vaccination rates increased significantly with the use of multivalent vaccines both at the ages of $12(p<0.001)$ and 24 months $(p<0.001)$. Finally, children whose parents expressed negative attitudes concerning vaccine cost, presented lower rates of timely immunization (statistically significant at the age of 24 months, OR: 0.51, 95\% CI: 0.28-0.92, $\mathrm{p}=0.025$ ).

Multiple logistic regression results assessing complete and age-appropriate immunization with all three newly introduced vaccines (Men C, PCV7, Var) are presented in Table 6. As shown, increasing age remained a negative predictor for complete (OR: 0.77; 95\% CI: 0.65-0.91, $\mathrm{p}=0.002$ ) and timely immunization at 12 (OR: 0.47 ; $95 \%$ CI: $0.35-0.64, p<0.001$ ) and 24 (OR: 0.45; 95\% CI: 0.29-0.71, $\mathrm{p}<0.001)$ months of age. Use of multivalent vaccines had a positive impact on age-appropriate immunization at 12 months (OR: 3.46; 95\% CI: 1.51-7.92, $\mathrm{p}=0.003$ ) while as increased household size ( $\geq 2$ children) was negatively associated with on time administration of vaccines (OR: 0.41; 95\% CI: 0.19-0.90, $\mathrm{p}=0.026$ ) at 24 months. Finally, children of mothers with higher academic education presented lower odds of age-appropriate immunization with all three newly introduced vaccines (OR: 0.42; 95\% CI: $0.19-0.94, \mathrm{p}=0.035)$ at 24 months. Results from univariate logistic regression models corresponding to the associations in Tables 5 and 6 are presented in as additional data [see Additional file 2: Tables S2 and S3] for comparability reasons.

\section{Discussion}

The present study provides important information regarding immunization coverage with traditional and new vaccines, as well as predictive factors for complete and ageappropriate vaccination of preschool children visiting public nurseries of the municipal district of Athens. Our results show that overall immunization rates and vaccination with primary series of traditional vaccines were satisfactory, except for hepatitis B, but the administration of booster doses was delayed. However, overall vaccination coverage was lower for newer vaccines, the most critical finding being the significant delay in administering the primary series of Men C and PCV7. The use of multivalent vaccines was a positive contributor in timely administration of all vaccines while a negative effect was noted with increasing household size. Child's increasing age was strongly associated with incomplete vaccination with all vaccines, while as immigrant status was a predictor of incomplete and delayed administration of all traditional vaccines, only. Finally, higher maternal education was associated with delayed administration of all newly licensed vaccines.

Regular assessment of vaccination coverage in a given country is a significant tool to improve the effectiveness of vaccination programs, but monitoring of vaccine uptake at a local level is also required in order to prevent vaccine preventable disease outbreaks due to susceptible subgroups [7,26-28]. Moreover, apart from monitoring coverage, it is necessary to identify characteristics or factors, associated with under- vaccination in order to develop appropriate strategies to increase compliance with immunization schedules [10,11,29].

According to our findings, rates of complete overall vaccination coverage of preschoolers against diphtheria, tetanus, pertussis, poliomyelitis, Haemophilus influenzae type b, as well as hepatitis B, were proven satisfactory, exceeding 94\%. As expected, overall uptake of Hib and 
Table 4 Number and weighted proportions for age- appropriate and complete immunization of the study population

\begin{tabular}{|c|c|c|c|c|}
\hline \multirow{4}{*}{ Vaccine } & \multicolumn{3}{|c|}{ Age appropriate immunization at: } & \multirow{3}{*}{$\begin{array}{l}\text { Complete immunization } \\
(n=731)\end{array}$} \\
\hline & 12 months & 24 months & 60 months & \\
\hline & $(n=731)$ & $(n=704)$ & $(n=56)$ & \\
\hline & N (\%) & N (\%) & $\mathrm{N}(\%)$ & $\mathrm{N}(\%)$ \\
\hline DTP/DTaP & $696(95.4)$ & $594(84.8)$ & $19(33.7)$ & $688(94,2)$ \\
\hline OPV /IPV & $629(86.8)$ & $602(86.2)$ & $22(38.8)$ & $723(98.9)$ \\
\hline $\mathrm{Hib}$ & $688(94.2)$ & $559(80.0)$ & $37(65.6)$ & $691(94.5)$ \\
\hline MMR & - & $660(90.5)$ & $20(35.9)$ & $462(63.7)$ \\
\hline HBV & $202(27.7)$ & $605(86.1)$ & $55(97.4)$ & $688(94.3)$ \\
\hline MenC & $164(22.9)$ & $111(16.1)$ & $7(12.4)$ & $671(91.9)$ \\
\hline PCV7 & $113(16.0)$ & $66(9.8)$ & $2(4.0)$ & $537(73.1)$ \\
\hline Var & - & $430(60.4)$ & $17(30.1)$ & $435(61.1)$ \\
\hline HAV & - & $6(0.9)$ & $41(71.7)$ & $450(62.0)$ \\
\hline All 5 traditional vaccines* (DTP/DTaP, OPV/ IPV, Hib, MMR, HBV) & $163(22.6)$ & $417(60.1)$ & $4(6.8)$ & $414(56.9)$ \\
\hline All 3 new* (PCV7, Var, MenC) & $62(8.8)$ & $35(5.2)$ & $1(2.0)$ & $327(44.2)$ \\
\hline
\end{tabular}

DTP/DTaP: diphtheria, tetanus, pertussis/ acellular pertussis vaccine; OPV/IPV: live attenuated/inactivated polio vaccine; HBV: hepatitis B vaccine; MMR: measles, mumps, rubella vaccine; Hib: haemophilus influenzae type b vaccine; Men C: conjugated meningococcal C vaccine; PCV7: conjugated pneumococcal 7-valent vaccine; Var: varicella vaccine; HAV: hepatitis A vaccine.

* For age-appropriate vaccination at 12 months, the MMR vaccine is excluded from the traditional vaccines denominator and the HAV is excluded from the new vaccines.

HBV was proven higher than recorded before $[19,21]$, possibly due to the widespread use and reimbursement of multivalent vaccines, along with the continuous training of primary care providers about the importance of early protection against hepatitis $\mathrm{B}$. Estimated vaccination coverage with the newer vaccines Men $\mathrm{C}, \mathrm{PCV} 7$, varicella, and HAV was lower, varying between 61 and $92 \%$, and this may be explained by the recent introduction of these vaccines in the Hellenic NIP and their even more recent full reimbursement policy, only since 2008 .

Age- appropriate immunization for older vaccines, except for HBV, was satisfactory; however, this was only true for primary series, with rates inversely associated as age advanced, indicating a delay in administration of booster doses. Efforts should be made to increase timely vaccination with the first dose of MMR, which did not exceed $90.5 \%$ at 24 months, and, even more so, to promote earlier administration of the second dose, as only $63 \%$ of our individuals had received their booster at the age of 5 years. The latter can be supported by the continuing periodic measles outbreaks in our country due to under immunized indigenous and immigrant population $[7,30]$. A remarkable result of this study, was the low rate of complete vaccination coverage against hepatitis $B$ before the age of 12 months (27.7\%), which has been a consistent finding in other studies in our country $[17,19,20]$. A possible explanation could be the lack of repayment of the hexavalent vaccine by the nation's largest social security fund (IKA), considering also that the majority of our population was insured with this fund.
In terms of the newest Men C, PCV7 and varicella vaccines, timely vaccination was also found to diminish with age. Nevertheless, the most striking finding was the unacceptably small percentage of children immunized by schedule against S. pneumoniae and $N$. meningitidis serogroup $C$, before the age of 12 months. This lack of compliance could be attributed to the high market price of the above vaccines and the unfavorable refund policy at the time of their introduction, pushing parents and caregivers to delay vaccination in order to reduce the number of required doses and therefore, cost. In contrast, on time vaccination against varicella and hepatitis A was higher, possibly as a result of the simultaneous NIP introduction with reimbursement.

Although achieving a high overall childhood vaccination coverage is important, on time administration of vaccine doses deserves special attention as delays may pose a significant risk for infectious diseases to young infants and children [31,32]. It has been emphasized that delayed administration of the first dose may jeopardize timely administration of the following vaccines [33]. Proposed solutions to address this problem include the creation of reminder systems through the mail or telephone, in addition to adequate training of, health workers and parents, even during pregnancy and the neonatal period $[26,33,34]$. In view of the recently introduced nationwide electronic drug prescription policy by all health professionals in our country, development of immunization registries appears feasible. Moreover, informing new parents in maternity hospitals about 
Table 5 Multiple logistic regression derived odds ratios (and 95\% Confidence Intervals) for the association between sample characteristics and immunization with all 5 traditional vaccines (DTP/DTaP, OPV/IPV, MMR, Hib, HBV)

\begin{tabular}{|c|c|c|c|c|c|c|}
\hline & \multirow{2}{*}{\multicolumn{2}{|c|}{$\begin{array}{l}\text { Complete immunization } \\
\qquad(n=731)\end{array}$}} & \multicolumn{4}{|c|}{ Age appropriate immunization at } \\
\hline & & & \multicolumn{2}{|c|}{12 months $(n=731)$} & \multicolumn{2}{|c|}{24 months $(n=704)$} \\
\hline & OR $(95 \% \mathrm{Cl})$ & $\mathrm{p}$ & OR $(95 \% \mathrm{Cl})$ & $\mathrm{p}$ & OR $(95 \% \mathrm{Cl})$ & $\mathrm{p}$ \\
\hline Actual age & $0.37(0.30-0.45)$ & $<0.001$ & $0.83(0.68-1.01)$ & 0.058 & $0.85(0.69-1.05)$ & 0.850 \\
\hline \multicolumn{7}{|l|}{ Father's nationality } \\
\hline Other & Reference & & & & & \\
\hline Greek & $1.56(1.04-2.36)$ & 0.034 & $0.98(0.61-1.59)$ & 0.930 & $2.57(1.71-3.87)$ & $<0.001$ \\
\hline \multicolumn{7}{|l|}{ Number of children } \\
\hline 1 & Reference & & & & & \\
\hline 2 & $1.02(0.69-1.50)$ & 0.932 & $1.64(1.03-2.62)$ & 0.038 & $0.95(0.63-1.42)$ & 0.796 \\
\hline $3+$ & $0.79(0.48-1.32)$ & 0.378 & $1.49(0.83-2.69)$ & 0.181 & $0.63(0.37-1.06)$ & 0.627 \\
\hline \multicolumn{7}{|l|}{ Maternal education } \\
\hline$\leq 12$ years & Reference & & & & & \\
\hline$>13$ years & $0.84(0.59-1.18)$ & 0.308 & $0.99(0.68-1.46)$ & 0.977 & $0.88(0.61-1.27)$ & 0.880 \\
\hline \multicolumn{7}{|l|}{ Use of multivalent vaccines } \\
\hline$\leq 4$-valent & Reference & & & & & \\
\hline 5- or 6-valent & $1.30(0.92-1.83)$ & 0.138 & $3.00(1.91-4.72)$ & $<0.001$ & $3.19(2.26-4.51)$ & $<0.001$ \\
\hline \multicolumn{7}{|c|}{ Impact of vaccine cost on the decision to vaccinate } \\
\hline No & Reference & & & & & \\
\hline Yes & $0.88(0.49-1.58)$ & 0.657 & $0.85(0.43-1.70)$ & 0.651 & $0.51(0.28-0.92)$ & 0.025 \\
\hline \multicolumn{7}{|c|}{ Impact of insurance reimbursement on the decision to vaccinate } \\
\hline No & Reference & & & & & \\
\hline Yes & $0.94(0.64-1.37)$ & 0.741 & $1.06(0.70-1.60)$ & 0.800 & $0.99(0.67-1.45)$ & 0.949 \\
\hline \multicolumn{7}{|l|}{ Place of vaccination } \\
\hline Privately & Reference & & & & & \\
\hline Insurance clinic / Public health clinic & $1.17(0.78-1.76)$ & 0.440 & $0.97(0.61-1.55)$ & 0.907 & $0.88(0.58-1.33)$ & 0.543 \\
\hline \multicolumn{7}{|l|}{ Type of insurance } \\
\hline IKA & Reference & & & & & \\
\hline Other & $0.97(0.63-1.48)$ & 0.876 & $1.39(0.87-2.22)$ & 0.165 & $1.26(0.82-1.94)$ & 0.296 \\
\hline
\end{tabular}

DTP/DTaP: diphtheria, tetanus, pertussis/ acellular pertussis vaccine; OPV/IPV: live attenuated/inactivated polio vaccine; HBV: hepatitis B vaccine; MMR: measles, mumps, rubella vaccine; Hib: haemophilus influenzae type $b$ vaccine.

IKA: Social Security Institute.

the importance of vaccination before discharge, could contribute significantly to timely administration of the first vaccine dose.

We found that the child's age remained a strong predictor for incomplete immunization with older and newer vaccines. Foreign ethnicity was significantly associated both with incomplete and delayed administration of all five traditional vaccines. Immigrant status as well as belonging to other minority groups (Roma, various religious groups) have been described as factors leading to suboptimal vaccination worldwide and, therefore, the need for targeted intervention in the aforementioned groups has been underlined $[21,35,36]$. Interestingly, in the present study, foreign born children presented lower vaccination rates only for traditional vaccines, when compared to native individuals, possibly indicating immunization trends in their countries of origin. In contrast, vaccination coverage with newer vaccines did not differ, and this could be indicative of improved compliance in a controlled environment of their receiving country. In light of the increasing inflow of refugee population from countries of poor socioeconomic status in Greece, the need for ongoing surveillance and targeted intervention is evident.

As described by other investigators $[21,37,38]$, increasing family size was associated with suboptimal, while as use of multivalent formulations with increased compliance for all vaccines, in our population. In addition, higher maternal education was a negative contributor to 
Table 6 Multiple logistic regression derived odds ratios (and 95\% Confidence Intervals) for the association between sample characteristics and immunization with all 3 newer vaccines (MenC, PCV7, Var)

\begin{tabular}{|c|c|c|c|c|c|c|}
\hline & \multirow{2}{*}{\multicolumn{2}{|c|}{$\begin{array}{l}\text { Complete immunization } \\
\qquad(n=731)\end{array}$}} & \multicolumn{4}{|c|}{ Age appropriate immunization at } \\
\hline & & & \multicolumn{2}{|c|}{12 months ( $n=731)$} & \multicolumn{2}{|c|}{24 months $(n=704)$} \\
\hline & OR $(95 \% \mathrm{CI})$ & $\mathrm{p}$ & OR $(95 \% \mathrm{Cl})$ & $\mathrm{p}$ & OR $(95 \% \mathrm{Cl})$ & $\mathrm{p}$ \\
\hline Actual age & $0.77(0.65-0.91)$ & 0.002 & $0.47(0.35-0.64)$ & $<0.001$ & $0.45(0.29-0.71)$ & 0.001 \\
\hline \multicolumn{7}{|l|}{ Father's nationality } \\
\hline Other & Reference & & & & & \\
\hline Greek & $1.29(0.88-1.90)$ & 0.188 & $2.10(0.91-4.86)$ & 0.082 & $2.95(0.91-9.50)$ & 0.071 \\
\hline \multicolumn{7}{|l|}{ Number of children } \\
\hline 1 & Reference & & & & & \\
\hline 2 & $0.91(0.64-1.31)$ & 0.621 & $0.58(0.31-1.09)$ & 0.092 & $0.41(0.19-0.90)$ & 0.026 \\
\hline $3+$ & $0.77(0.48-1.22)$ & 0.265 & $0.42(0.18-1.01)$ & 0.053 & $0.27(0.08-0.89)$ & 0.031 \\
\hline \multicolumn{7}{|l|}{ Maternal education } \\
\hline$\leq 12$ years & Reference & & & & & \\
\hline$>13$ years & $0.73(0.53-1.02)$ & 0.061 & $0.68(0.38-1.22)$ & 0.198 & $0.42(0.19-0.94)$ & 0.035 \\
\hline \multicolumn{7}{|l|}{ Use of multivalent vaccines } \\
\hline$\leq 4$-valent & Reference & & & & & \\
\hline 5- or 6-valent & $1.09(0.78-1.50)$ & 0.630 & $3.46(1.51-7.92)$ & 0.003 & $2.24(0.88-5.68)$ & 0.090 \\
\hline \multicolumn{7}{|c|}{ Impact of insurance reimbursement on the decision to vaccinate } \\
\hline No & Reference & & & & & \\
\hline Yes & $0.95(0.67-1.34)$ & 0.764 & $1.22(0.65-2.28)$ & 0.538 & $1.28(0.57-2.90)$ & 0.551 \\
\hline \multicolumn{7}{|l|}{ Place of vaccination } \\
\hline Privately & Reference & & & & & \\
\hline Insurance clinic / Public health clinic & $1.39(0.95-2.03)$ & 0.086 & $0.64(0.32-1.30)$ & 0.216 & $0.56(0.23-1.36)$ & 0.199 \\
\hline \multicolumn{7}{|l|}{ Type of insurance } \\
\hline IKA & Reference & & & & & \\
\hline Other & $1.13(0.77-1.66)$ & 0.545 & $1.67(0.84-3.34)$ & 0.144 & $1.69(0.70-4.11)$ & 0.244 \\
\hline
\end{tabular}

Men C: conjugated meningococcal C vaccine; PCV7: conjugated pneumococcal 7-valent vaccine; Var: varicella vaccine.

IKA: Social Security Institute.

timely immunization with new vaccines. This was in accordance with a number of reports describing that parents of higher educational level may present lower compliance to vaccine recommendations as a result of their access to various information sources, increasing their concern about vaccine safety [39-41].

Finally, we were not able to draw any firm conclusions on the negative impact of cost on the decision of parents to vaccinate their children. Nevertheless, the increasing proportions of vaccination coverage among our study population in comparison to previously described rates [19] indicates that insurance reimbursement had a positive impact, a conclusion also validated by our model findings, although not to a statistically significant degree. The insignificant impact of cost on immunization in our study may be partly explained by the small percentage of guardians responding positively to the question of whether cost would be a deciding factor for them (8.3\%), but also by the retrospective collection of information, comparing current parental beliefs towards vaccines that had already been administered. However, the importance of state's funding policy has been clearly associated with vaccination coverage in a number of studies, as out-of- pocket costs have been inversely correlated with optimal vaccine administration [42-45]. In view of the economic crisis in our country, an increasing number of children have no insurance for recommended vaccines and visit, therefore, public health centers to receive immunization. In order to prevent immunization gaps, it is essential to improve vaccine delivery in public facilities, including schools, as well as maintaining full reimbursement of all necessary vaccines.

\section{Study limitations and strengths}

The present study had a number of limitations. It was conducted locally, among families of low and middle income and is, therefore, not representative of the whole country. Moreover, the municipal nurseries of Athens 
could be considered as a supervised environment since the immunization records of all students are tested upon registration and yearly thereafter. Although this could overestimate vaccination coverage, it must be emphasized that non- compliance to complete immunization gaps is not a criterion to deny school entry. Nevertheless, the above article provides significant evidence regarding immunization, collected accurately through health booklets, in the nation's largest city hosting the majority of native and immigrant population. Therefore, it could be used as a model for designing strategies in order to improve quality and effectiveness of vaccination programs on a local basis.

\section{Conclusions}

Our results highlight the need to monitor uptake of newly introduced vaccines and to develop an effective vaccine reminder system, in order to encourage appropriate administration of booster doses, as well as timely administration of hepatitis B vaccine. Considering the recent introduction of a nationwide electronic drug prescription policy, the implementation of immunization registries in order to improve the quality of vaccination programs, appears feasible. Immigration, increasing number of siblings and high maternal education were factors associated with suboptimal immunization and may warrant targeted intervention. The support of public health facilities, including schools, as well as the maintenance of a full reimbursement policy, are of paramount importance as gaps in insurance benefits, in view of the economic crisis, could result in missed vaccination opportunities in our country.

\section{Additional files}

Additional file 1: Table S1. 2006 National immunization programme recommendations.

Additional file 2: Table S2. Univariate logistic regression derived odds ratios (and 95\% Confidence Intervals) for the association between sample characteristics and immunization with all 5 traditional vaccines (DTP/DTaP, OPV/IPV, MMR, Hib, HBV). Table S3. Univariate logistic regression derived odds ratios (and 95\% Confidence Intervals) for the association between sample characteristics and immunization with all 3 newer vaccines (MenC, PCV7, Var).

\section{Competing interests}

The authors declare that they have no competing interest.

\section{Authors' contributions}

IDP: study conception and design, drafting of manuscript, critical revisions for important intellectual content, supervision, KAM: study design, data collection, drafting of manuscript, ES: statistical expertise, data analysis, critical revisions GT: data collection, technical support, KT: supervision, administrative support. All authors read and approved the final manuscript.

\section{Acknowledgements}

The authors would like to thank Ms C. loannidou, RN, MSc for technical and material assistance, nursery teachers for their support and all parents for participating in this study.

\section{Funding}

This research was partially funded by Vianex/ Sanofi Pasteur MSD, Vaccine Unit, Greece.

\section{Author details}

${ }^{1}$ Pediatric Clinic, "P \& A Kyriakou" Children's Hospital, National and Kapodistrian University of Athens, Faculty of Nursing, Athens, Greece. ${ }^{2}$ Postgraduate Program, National and Kapodistrian University of Athens, Faculty of Nursing, Athens, Greece. ${ }^{3}$ Department of Hygiene, Epidemiology and Medical Statistics, National and Kapodistrian University of Athens Medical School, Athens, Greece. ${ }^{4}$ Department of Preventive Medicine, Municipal Nursery of Athens, Athens, Greece

Received: 8 January 2013 Accepted: 25 September 2013

Published: 2 October 2013

\section{References}

1. American Academy of Pediatrics: Active and Passive Immunization. In Red Book: 2012 Report of the Committee in Infectious Diseases. Edited by Pickering LK, Baker CJ, Kimberlin DW, Long SS. Elk Grove Village, IL: American Academy of Pediatrics; 2012:1-2.

2. European Centre for Disease Prevention and Control: Annual Epidemiological Report 2011. Reporting on 2009 surveillance data and 2010 epidemic intelligence data. Stockholm: ECDC; 2011

3. Centers for Disease Control and Prevention (CDC): Progress in Global Measles Control, 2000-2010. MMWR Morb Mortal Wkly Rep 2012, 61:73-75.

4. Centers for Disease Control and Prevention (CDC): Global Routine Vaccination Coverage, 2010. MMWR Morb Mortal Wkly Rep 2011, 60(Suppl 44):1520-1522.

5. Anis E, Grotto I, Moerman L, Warshavsky B, Slater PE, Lev B, Israeli A: Measles in a highly vaccinated society: the 2007-08 outbreak in Israel. J Infect 2009, 59(1522Suppl 4):252-258.

6. Lopalco PL, Martin R: Measles still spreads in Europe: who is responsible for the failure to vaccinate? Euro Surveill 2010, 15(Suppl17):pii 19557.

7. Pervanidou D, Horefti E, Patrinos S, Lytras T, Triantafillou E, Mentis A, Bonovas S, Panagiotopoulos T: Spotlight on measles 2010: ongoing measles outbreak in Greece, January-July 2010. Euro Surveill 2010, 15(Supl 30):pii 19629.

8. Centers for Disease Control and Prevention (CDC): Notes from the field: Measles outbreak - Indiana, June - July 2011. MMWR Morb Mortal Wkly Rep 2011, 60(Suppl 34):1169.

9. Walker J, Huc S, Sinka K, Tissington A, Oates K: Ongoing outbreak of mumps infection in Oban, Scotland, November 2010 to January 2011. Euro Surveill 2011, 16(Suppl 8):pii 19803.

10. Falagas ME, Zarkadoulia E: Factors associated with suboptimal compliance to vaccinations in children in developed countries: a systematic review. Cur Med Res Opin 2008, 24(Suppl 6):1719-1741.

11. Torun SD, Bakirci N: Vaccination coverage and reasons for non-vaccination in a district of Instanbul. BMC Public Health 2006, 6:125-132.

12. Muhsen K, Abed El-Haia R, Amit-Aharonb A, Nehamab H, Gondiaa M, Davidovitchc N, Gorena S, Cohena D: Risk factors of underutilization of childhood immunizations in ultraorthodox Jewish communities in Israel despite high access to health care services. Vaccine 2012, 30:2109-2115.

13. Ughade SN, Zodpey SP, Deshpande SG, Jain D: Factors responsible for delayed immunisation among children under 5 years of age. J Indian Med Assoc 2000, 98(Suppl 1):4-5.

14. Abbotts B, Osborn LM: Immunization status and reasons for immunization delay among children using public health immunization clinics. Am J Dis Child 1993, 147(Suppl 9):965-968.

15. Vandermeulen C, Roelants M, Theeten H, Van Damme P, Hoppenbrouwers K: Vaccination coverage and sociodemographic determinants of measles-mumps-rubella vaccination in three different age groups. Eur J Pediatr 2008, 167(Suppl 10):1161-1168.

16. Prislin R, Dyer JA, Blakely CH, Johnson CD: Immunization status and sociodemographic characteristics: the mediating role of beliefs, attitudes, and perceived control. Am J Public Health 1998, 88(Suppl 12):1821-1826.

17. Gavana M, Tsoukana P, Giannakopoulos E, Smirnakis E, Mpenos A: Adequacy of vaccination coverage at school-entry: Cross-sectional study in schoolchildren of an urban population. Arch Hellen Med 2005 22(Suppl 4):358-369. article in Greek. 
18. Lionis C, Chatziarsenis M, Antonakis N, Giannoulis Y, Fioretos M: Assesment of vaccine coverage of schoolchildren in three primary health care areas in rural Crete, Greece. Fam Pract 1998, 15:443-448.

19. Pavlopoulou ID, Michael K, Tsoumakas C, Tsiftis G, Theodoridou M, Androulakis I: Trends in immunization coverage among preschool children in Athens, Greece, following the introduction of new vaccines [abstract]. In 5th World Congress of the World Society for Pediatric Infectious Diseases-WSPID, 15-18 November 2007. Bangkok: WSPID; 2007:238.

20. Panagiotopoulos T, Valassi- Adam E, Sarafidou E, Mandeki A, Stratiki Z, Benos A, Adamidis D, Koutis A, Lionis C: Greek study on vaccination coverage. Arch Hellen Med 1999, 16(Suppl 2):154-162. article in Greek.

21. Danis K, Georgakopoulou T, Stavrou T, Laggas D, Panagiotopoulos T: Socioeconomic factors play a more important role in childhood vaccination coverage than parental perceptions: a cross - sectional study in Greece. Vaccine 2010, 28:1861-1869.

22. Mediterranean Migration Observatory: Statistical Data on Immigrant in Greece: An analytic study of available data and recommendations for conformity with European Union Standards. [http://www.mmo.gr/pdf/ general/IMEPO_Final_Report_English.pdf].

23. Hellenic Statistical Authority: 2001 Census Data. [http://www.statistics.gr]

24. Snedecor GW, Cochran WG: Statistical Methods. 7th edition. Ames, lowa: lowa State University Press; 1980.

25. Mc Cullagh P, Nedler JA: Generalized Linear Models. New York, NY: Chapman and Hall, Inc; 1989.

26. Haverkate M, D'Ancona F, Johansen K, Van Der Velden K, Giesecke J, Lopalco PL: Assessing vaccination coverage in the European Union: is it still a challenge? Expert Rev Vaccines 2011, 10(Suppl 8):1195-1205.

27. Guerin N, Roure C: Immunization coverage in the European Union. Euro Surveill 2007, 2(1):185.

28. Hahne S, Mayce J, van Binnendijk R, Kohl R, Dolman S, van der Veen $Y$, Tipples G, Ruijs H, Mazzulli T, Timen A, van Loon A, de Melker H: Rubella outbreak in The Netherlands, 2004-2005: high burden of congenital infection and spread to Canada. Pediatr Infect Dis J 2009, 28(9):795-800.

29. Bates AS, Wolinsky FD: Personal, financial, and structural barriers to immunization in socioeconomically disadvantaged urban children. Pediatrics 1998, 101(4 Pt 1):591-596.

30. Georgakopoulou T, Grylli C, Kalamara E, Katerelos P, Spala G, Panagiotopoulos T: Current measles outbreak in Greece. Euro Surveill 2006, 11(2), E060223.2.

31. Grant CC, Roberts M, Scragg R, Stewart J, Lennon D, Kivell D, Ford R, Menzies R: Delayed immunisation and risk of pertussis in infants: unmatched case-control study. BMJ 2003, 326(7394):852-853.

32. Kolos $V$, Menzies $R$, Mclntyre P: Higher pertussis hospitalization rates in indigenous Australian infants, and delayed vaccination. Vaccine 2007, 25(4):588-590

33. Petoussis- Harris H, Cameron CG, Goodyear- Smith F, Turner N, York D, Jones $\mathrm{R}$, Stewart J: What contributes to delays? The primary care determinants of immunization timeliness in New Zealand. J Prim Health Care 2012, 4(1):12-20.

34. Linkins RW, Dini EF, Watson G, Patriarca PA: A randomized trial of the effectiveness of computer-generated telephone messages in increasing immunization visits among preschool children. Arch Pediatr Adolesc Med 1994, 148(9):908-914.

35. Buelow VH, Van Hook J: Timely Immunization series completion among children of immigrants. J Immigr Minor Health 2008, 10:37-44.

36. Poethko-Mueller C, Ellert U, Kuhnert R, Neuhauser $H$, Schlaud M, Schenk L: Vaccination coverage against measles in German - born and foreign - born children and identification of unvaccinated subgroups in Germany. Vaccine 2009, 27:2563-2569.

37. Samad L, Tate AR, Dezateux C, Peckham C, Butler N, Bedford H: Differences in risk factors for partial and no immunisation in the first year of life: prospective cohort study. BMJ 2006, 332(7553):1312-1323.

38. Koslao-Petraco MB, Judelson RG: Societal impact of combination vaccines: experiences of physicians, nurses and parents. J Pediatr Health Care 2008, 22:300-309.

39. Dannetun E, Tegnell A, Hermansson G, Törner A, Giesecke J: Timeliness of MMR vaccination-influence on vaccination coverage. Vaccine 2004 , 22(31-32):4228-4232.

40. Hak E, Schonbeck Y, De Melker H, Van Essen GA, Sanders EAM: Negative attitude of highly educated parents and health care workers towards future vaccinations in the Dutch childhood vaccination program. Vaccine 2005, 23:3103-3107.
41. Poethko-Müller C, Kuhnert R, Schlaud M: Vaccination coverage and predictors for vaccination level. Results of the German Health Interview and Examination Survey for Children and Adolescents (KiGGS). Bundesgesundheitsblatt Gesundheitsforschung Gesundheitsschutz 2007 50(5-6):851-862. article in German.

42. Davis MM, Ndiaye SM, Freed GL, Kim CS, Clark SJ: Influence of insurance status and vaccine cost on physicians' administration of pneumococcal conjugate vaccine. Pediatrics 2003, 112(Suppl 3):521-526.

43. Hinman AR, Orenstein WA, Rodewald L: Financing immunizations in the United States. Clin Infect Dis 2004, 38(10):1440-1446.

44. Molinari N-AM, Kolasa M, Messonier M, Schieber RA: Out-of-pocket costs of childhood immunizations: A comparison by type of insurance plan. Pediatrics 2007, 120(5):e1148-e1156.

45. Smith PJ, Molinari NA, Rodewald L: Underinsurance and pediatric immunization delivery in the United States. Pediatrics 2009, 124(5):S507-s514.

\section{doi:10.1186/1471-2458-13-908}

Cite this article as: Pavlopoulou et al: Immunization coverage and predictive factors for complete and age-appropriate vaccination among preschoolers in Athens, Greece: a cross- sectional study. BMC Public Health 2013 13:908.

\section{Submit your next manuscript to BioMed Central and take full advantage of:}

- Convenient online submission

- Thorough peer review

- No space constraints or color figure charges

- Immediate publication on acceptance

- Inclusion in PubMed, CAS, Scopus and Google Scholar

- Research which is freely available for redistribution

Submit your manuscript at www.biomedcentral.com/submit
C) Biomed Central 